\title{
Violencia de género, feminismo y representación en Cuba
}

\author{
Yuliuva Hernández García (iD 0000-0002-1879-9637 \\ Instituto Superior Minero Metalúrgico de Moa, Holguín, Cuba. 83300 - \\ webmaster@ismm.edu.cu
}

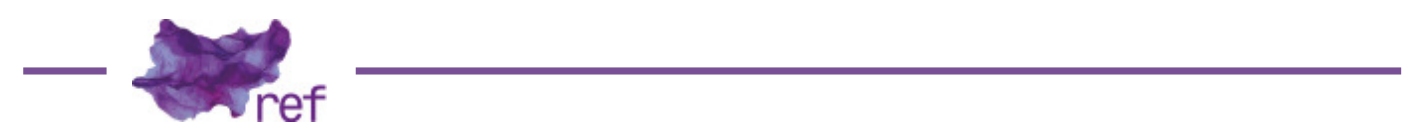

Resumen:El artículo presenta un análisis teórico-metodológico de los estudios sobre violencia contra las mujeres por motivos de género en Cuba, problemáticas que presenta el feminismo cubano actual y los vínculos de estas cuestiones con el fenómeno de la representación, tomando como referente para el debate y crítica las investigaciones sobre el tema por más de diez años en el municipio Moa. El análisis se realiza sobre la base teórica y metodológica del feminismo descolonial, en su especial interrelación con las Epistemologías del Sur, para ofrecer otras posibles alternativas de transformación para la ciencia y el activismo feministas cubanos, los estudios sobre violencia en específico y la práctica política feminista en general en los actuales momentos que vive el país.

Palabras clave: violencia contra mujeres; feminismo; representación; feminismo descolonial; epistemologías del sur

\section{Gender Violence, Feminism and Representation in Cuba}

Abstract: The article presents a theoretical and methodological analysis of studies on gender-based violence against women in Cuba, problems presented by current Cuban feminism and the connections between both issues and the phenomenon of representation, taking as a reference for debate and criticism investigations that have been made for over ten years on the subject in the municipality of Moa. The analysis is carried out based on the decolonial feminism, in its special interrelationship with the Epistemologies of the South, to offer other possible alternatives of transformation for Cuban feminist science and activism, studies on gender-based violence against women in particular and feminist political practice feminist in general in today's Cuba.

Key-word: violence against woman; feminism; representation; decolonial feminism; epistemologies of the South

\section{Introducción}

Cada vez que se conoce algún caso de violencia de género en Cuba, ya sea por estadísticas o por rumores de la gente que vive en este espacio nacional y cotidiano que se habita más allá de La Habana y de las capitales provinciales cubanas -espacios cargados de múltiples desigualdades socio-territoriales acumuladas por décadas-, analizo hasta qué punto las violencias y vivencias experimentadas por tantas mujeres política, geográfica y subjetivamente distantes de los centros de enunciación del feminismo cubano actual han de ser parte de esta lucha y estos estudios que por años se han desarrollado en el país; hasta qué punto la representación (Gayatri SPIVAK, 2009; Mario RUFER, 2012; 2017) por parte de otras y otros, muchas veces feministas y con buenas intenciones convertidos en los sujetos del discurso y de la enunciación, representa realmente tanta diversidad y problemas sociales.

En ese contexto, este trabajo tiene el objetivo de reflexionar sobre la violencia de género en Cuba, el feminismo cubano, y el problema de la representación, tomando como base teórica al Feminismo Descolonial y las Epistemologías del Sur; así como, de la práctica, la investigación sostenida como un estudio de caso por muchos años sobre el actual fenómeno de la violencia de 
género en Moa (Cuba). Se reflexiona acerca del actual accionar teórica y metodológicamente extractivista de gran parte del feminismo académico/activista cubano, pensando de qué manera se puede contribuir al análisis y transformación de la violencia de género contra mujeres, niñas, niños y adolescentes desde una visión que incorpore -en sentido contrario a lo existentemetodologías otras.

\section{Feminismo cubano post 1959 y violencia de género: análisis histórico-descolonial}

El feminismo cubano tiene una larga historia de luchas y conquistas. Sin embargo, para los Estudios de Género y la práctica políica feminista en Cuba, parece haber un antes y un después. La realidad social se nos presenta hoy cambiante en contenidos y formas, aflorando prácticas políticas, investigativas y académicas que reproducen la colonialidad del saber-poder (Aníbal QUIJANO, 2007), a la vez que no dan cuenta de la insospechada diversidad de realidades otras en Cuba hoy, incluidas las de género.

Tales prácticas se producen desde una ciencia que se dice feminista, pero que en general aún se autodeleita en conceptos y teorías importados, se autopublica y visibiliza sin haber llegado antes hasta otros contextos reales de estudio, en tanto se gesta desde las presunciones de un único punto de vista -el citadino-habanero o metropocéntrico-, deviniendo en aquello que autores descoloniales de América Latina han identificado hace tiempo como hybris del punto cero (Santiago CASTRO-GÓMEZ, 2007) para caracterizar el tipo de modelo epistémico preponderante en el mundo occidental: aquel que observa (investiga) sin ser observado (lo cual impide la impugnación) y ello deviene en garantía de la objetividad científica y legitimidad.

Desde la subalternidad (SPIVAK, 2009) y las márgenes (Chandra T. MOHANTY, 2008) se producen miradas y lecturas solo accesibles al (la) subalterno (a) como grupo social o como actor social individual, en un contexto de relaciones de poder. Por ello, en un acto de lealtad a las pertenencias y a una ciencia feminista más democrática, afín con las epistemologías feministas descoloniales del Sur, hago explícitos mis lugares de enunciación e inquietudes, en un contexto académico que valoro como colonialista: mujer, blanca, investigadora joven y oriental de un municipio cubano (Moa, en Holguín). Todo ello tiene su impronta en mis lecturas de las luchas feministas de hoy en Cuba y en el devenir de lo que acá se auto-asume como ciencia feminista.

Entiendo que las históricas luchas feministas y los actuales Estudios de Género en Cuba se hayan indisolublemente ligados a la figura de Vilma Espín, en la dirección por muchos años de la Federación de Mujeres Cubanas (FMC); lo que podría identificarse como un movimiento social en sus inicios, por su activismo pujante al triunfo revolucionario de 1959, hasta la década de los noventa del siglo XX, pero que fue declinando hasta prácticamente perder en la actualidad todo su protagonismo social en las luchas de las mujeres cubanas, con situaciones de desigualdad cada vez más complejas hoy. En aquellos momentos iniciales, a pesar de la diversidad de las mujeres en el país, todas se sentían en general protagonistas y parte de una sociedad más justa, inclusiva, que se encaminaba a erradicar las desigualdades por conceptos de género: mujeres en Revolución. En gran medida se logró, porque las mujeres cubanas han gozado de privilegios pocas veces conocidos por otras en el mundo; aunque no exactamente todas fueron incluidas por igual, sobre todo las del grupo de la sexo-diversidad.

Aun cuando pueda objetársele cualquier desacierto, con Vilma se construye una causa de las mujeres cubanas post-Revolución, asociada al internacionalismo y a la lucha contra el capitalismo global. Junto a su dirección se diseña y abre el primer programa de Maestría en Cuba en Estudios de Género en 2005 en la Universidad de La Habana que, aunque rezagado con respecto al mundo, significaba un paso de avance desde el punto de vista científico y feminista. Ese primer acercamiento avalado por la FMC y su dirección nacional, que significó estudiar el feminismo internacional, implicaba haber reconocido que con la Revolución, al pasar los años, no se había logrado desmantelar un problema fundamental que seguía en la base de muchos problemas de las mujeres cubanas, el patriarcado. Por lo tanto, sí había que investigar y tratar de cambiar las realidades. Para ese entonces, a pesar de la crisis socioeconómica atravesada en los noventa, no se imaginaban las dimensiones que se habían ido regenerando de la desigualdad de género y social.

Y esto fue así porque, precisamente una de las mayores críticas realizadas al feminismo marxista/socialista-del cual nuestro movimiento era parte- fue haber subsumido y postergado los problemas específicos de las mujeres por la causa más general y urgente que eran las luchas de clase del proletariado (Judith ASTELARRA, 2005). Durante mucho tiempo no se ha considerado necesario pensar en profundidad la política con enfoque de género (más allá de la frase mujeres en Revolución), creyendo que la Revolución social automáticamente nos incluyó a todos por igual y se eliminaron las inequidades de todo tipo.

Dentro de ese panorama socio-político, se instauró en una suerte de verdad, el discurso de que en Cuba no había -por ejemplo- violencia contra mujeres, niñas y adolescentes, al punto de 
que aún hoy no se encuentran fácilmente datos y con carácter público sobre la violencia de género en el país; a pesar de ser parte de los Estados firmantes de la CEDAW y otras muchas acciones feministas internacionales actuales. Entre las múltiples consecuencias asociadas a este fenómeno, se halla la carencia de políticas públicas eficaces para la identificación y el tratamiento social particularizado de la violencia contra las mujeres por motivos de género: al restar importancia al problema, se han generado históricamente grandes subregistros estadísticos del mismo y desatención.

No se ha logrado aún la implementación de una normativa legal severa en el sistema penal cubano para el tratamiento de la violencia de género, ni la creación de instituciones de acogida a mujeres víctimas con sus hijos. El tratamiento a las víctimas y a los agresores no está declarado como programa en las instituciones de salud-como por ejemplo los Centros Comunitarios de Salud Mental- y el trabajo que se realiza tiene lugar en lo fundamental en las capitales provinciales, ya que en las comunidades alejadas de los centros urbanos cubanos la realidad es otra por el trabajo desacertado, empírico y sin preparación de la FMC en esos espacios (Yuliuva HERNÁNDEZ, 201 1, 2013b, 2014a; Alisa DELGADO; HERNÁNDEZ, 201 1; HERNÁNDEZ; DELGADO, 2014b, 2016).

Hoy, leyendo la realidad cubana y las luchas de las mujeres desde un pensamiento feminista descolonial, considero que tras el paso de casi 30 años de la introducción del tema en la academia, lo cierto es que mientras en Latinoamérica, Asia y África se lucha por descolonizar los Estudios de Género y las ciencias sociales en general, en Cuba en la mayor parte de los estudios y eventos teóricos se siguen reproduciendo los colonizadores paradigmas de interpretación nor-eurocéntricos (MOHANTY, 2008), incluyendo el pensamiento marxista, que dejan fuera a muchas mujeres cubanas reales y diferentes, atravesadas por múltiples interseccionalidades en medio de cambios y crisis.

Puedo afirmar, porque fui alumna de ese primer programa de estudios en 2005 y he seguido investigando hasta la actualidad, que las luchas feministas y las comprensiones de las teorías de género han quedado rezagadas. Ello ha redundado en las más variadas investigaciones que enuncian trabajar desde la categoría género, pero solo se hallan en el enunciado descriptivo, o en datos estadísticos desagregados por sexo, o en los diseños y/o implementación de proyectos asistidos por la Cooperación Internacional como una de las variables incluidas para poder clasificar y recibir financiamientos. No se ha conocido en profundidad el excelente trabajo de las autoras feministas descoloniales, del feminismo negro, del indígena, del transnacional, del nuestro americano para comprender las desigualdades y los problemas más presentes hoy en nuestras vidas como mujeres.

Para valorar con mayor grado de precisión este asunto, aunque la colonialidad académica en la diada Cuba-Mundo se produce en casi todas las problemáticas de género que se estudian, me remito a lo que forma parte de mis mayores acercamientos científicos desde la Sociología: la violencia contra las mujeres por motivos de género. De una etapa inicial en la década de los noventa del siglo XX hasta inicios del 2000, caracterizada por trabajos enfáticos en mostrar las cifras de violencia contra las mujeres según las diversas tipologías que se producían en algunos lugares en Cuba, considero que aún no se trasciende a comprensiones teóricas otras del problema en el particular contexto cubano (HERNÁNDEZ, 2014a). La mayor parte de los estudios se producen, fundamentalmente, reproduciendo teorías hegemónicas y construyendo estadísticas muy limitadas sobre violencia. En las compilaciones de artículos, se aprecia poca presencia de investigadoras(es) no habaneras, así como análisis de situaciones con escasa profundidad teórica y metodológica, de manera que puedan ponerse al debate las teorías legitimadas desde ese feminismo noreurocentrado. Las pocas publicaciones sobre el tema lo hacen palpable (CIPS' 2010, 2011 a, 2011 b; Iyamira HERNÁNDEZ, 2014c) ${ }^{2}$

Sobre esta cuestión del feminismo cubano, Mayda Álvarez Suárez, Directora del Centro de Estudios de la Mujer, que pertenece al nivel nacional de la FMC, señala:

Aún son escasos los estudios que aborden críticamente las diferentes corrientes teóricas en torno al problema de género. La bibliografía de autoras y autores nacionales es utilizada cada vez más, pero observamos que se extrapolan análisis que correspondieron a un momento y lugar diferente, se generalizan y tal como ocurre con las clásicas, los conceptos no son interpelados y resignificados según los contextos y situaciones específicas. Por otra parte, no se ha incorporado al debate nacional el rico intercambio, no exento de contradicciones, que se ha desarrollado en el plano internacional en torno a los conceptos de género, no se observa suficiente actualización sobre otras teorías que se han abierto camino en otras latitudes. Por tanto se reproducen en las investigaciones aspectos como identificar género con mujer, aferrarse a una lectura binaria del análisis de género al identificarlo sólo como diferencia sexual. Se impone la necesidad de estudiar los cambios que se producen en la actualidad de manera

\footnotetext{
Centro de Investigaciones Psicológicas y Sociológicas (CIPS).

2 Ver también la producción de libros de la FMC a través de la Editorial de la Mujer, por ejemplo Género. Teoría o Violencia. Hacerla visible, ambos del 2008.
} 
diferenciada, según la inserción en las diferentes personas a la sociedad, sus edades, color de la piel, territorio en el que viven y otras variables diferenciadoras (ÁLVAREZ, 2016, p. 6).

Estas cuestiones, recientemente reconocidas, han implicado múltiples problemáticas; entre ellas, las universalizaciones acerca de la violencia, hoy devenidas en esencialismos dentro del propio contexto cubano acerca de un sujeto mujer víctima homogéneo, que en general ignora las complejidades de una multiplicidad de mujeres en toda la nación, que habitan realidades a veces insospechadas por investigadoras(es), como resultado -sobre todo- de una creencia naturalizada durante mucho tiempo en el país sobre la inexistencia de fenómenos como la pobreza o la violencia cruenta contra mujeres, toda vez que el proceso revolucionario instituyó en los imaginarios sociales la desarticulación per se de las injusticias e inequidades.

Otro problema para la explicación de la violencia contra mujeres por motivos de género en Cuba, ha sido la falta de comprensión como un fenómeno atravesado por múltiples condicionantes, entre las cuales las culturas patriarcales cubanas de espacios geográficos específicos juegan un papel fundamental. De ello se desprende que, a diferencia de la investigación en contextos como el latinoamericano donde existen estudios sobre lo que ha llegado a los extremos de femi-genocidio y se articulan nuevas visiones entre cultura-violencia-cuerpos-espacio-territorio-guerra-crimen organizado (Karina BIDASECA, 2013, 2015; Rita SEGATO, 2012, 2013), en Cuba los análisis son pocos y se ha carecido de estudios sobre cómo opera la violencia contra las mujeres en los marcos de nuestros también diversos contextos socioculturales, no obstante las situaciones de violencia de género no se hallen mediadas por la guerra, el tráfico de drogas, las mafias o los males sociales que azotan a Latinoamérica u otras partes del mundo.

Esta situación tiene otro correlato, y es lo que considero equivalente a la colonización discursiva ${ }^{3}$ (MOHANTY, 2008), en la otra diada que se produce: Cuba-Cuba o Cuba-Provincias. Me refiero al correlato de la supuesta representación: la autorizada voz occidental (habanera, o metropocéntrica cuando no habanera) que habla por las otras voces, las subalternas. Con tan solo analizar las pocas publicaciones en Cuba sobre los temas de género, es posible identificar el fenómeno de la invisibilidad de las ya existentes y reconocidas académicas feministas en el resto de las universidades e instituciones científicas del país. En el caso específico de los estudios sobre violencia de género, una sencilla muestra del fenómeno lo constituyen los Boletines en Internet No a la violencia que publica el Servicio de Noticias de la Mujer Latinoamericana y Caribeña (SEMlac) y se circula a través de correo electrónico, mediante un directorio que posee de aquellas (os) que trabajan el tema en Cuba.

Este modo de supuesta representación por parte de las académicas feministas cubanas visibles, en nombre de los Estudios de Género y las mujeres, tiene que ver con lo que explica Yuderkis Espinosa:

(...) un buen ejemplo de lo que he denominado racismo del género: una imposibilidad de la teoría feminista de reconocer su lugar de enunciación privilegiada dentro de la matriz moderna colonial de género, imposibilidad que se desprende de su negación a cuestionar y abandonar este lugar a costa de "sacrificar", invisibilizando diligentemente, el punto de vista de "las mujeres" en menor escala de privilegio (ESPINOSA, 2014, p. 10).

¿Qué validez ha tenido entonces el género como categoría analítica para generar una ciencia feminista, cambios en nuestros paradigmas científicos y, en consecuencia, en las políticas públicas que atañen a los problemas de las relaciones de género? Entiendo que el potencial inicial era bastante para transformar las realidades de las cubanas, no obstante haber importado teorías, conceptos, enfoques, pues quedaba abierta la posibilidad de un desarrollo ulterior propio. Pero las circunstancias sociopolíticas nacionales e internacionales, junto a las prioridades $y$ urgencias de la Revolución antes, y de la sociedad cubana hoy en pleno proceso de replanteamiento como modelo socio-económico, han menguado el empuje que se gestaba a nivel social y en las agendas científicas.

O, si se gesta un desarrollo, es parcial al seguir produciéndose en determinados espacios que continúan siendo dominantes como la capital cubana. Como categoría analítica ha puesto al descubierto problemáticas que ni siquiera se sospechaban; pero ello no ha significado un punto de giro en las teorías que generara un corpus con identidad propia posibilitado de explicar nuestra unidad y nuestra diferencia, de forma tal que pudiera romper-desde la dialéctica-con los conceptos y teorías nor-eurocéntricos asumidos muchas veces acríticamente de otros escenarios. Una de las causas que han conducido a ello, ha sido precisamente el alejamiento de las

\footnotetext{
${ }^{3}$ Chandra Mohanty (2008) plantea una propuesta epistemológica de descolonizar el conocimiento y develar la manera en que las representaciones textuales de aquellos sujetos sociales -construidos como "los otros" en distintos contextos geográficos e históricos- se convierten en una forma de colonialismo discursivo que no sólo da cuenta de una realidad sino que la construye. La importancia de visibilizar estos efectos del colonialismo discursivo es patente, sobre todo cuando vemos su tenaz presencia en las representaciones de los Otros/as en la investigación teórica y en la intervención. No desvelar estas "jugadas del colonialismo" es contraproducentes para los objetivos del feminismo como ideario político global y multicultural.
} 
académicas feministas de las realidades de las mujeres del resto del país, circunscribiendo sus estudios a los entornos más próximos a ellas y luego universalizando sobre un "sujeto mujer" homogéneo en Cuba.

Ante toda esta realidad, no observada desde la hybris del punto cero que representan las académicas feministas cubanas del escenario dominante, cuyo lugar de enunciación está siempre sobre el privilegio del punto de vista; realidad que no ha generado una preocupación justa para la FMC hasta hace solo muy poco tiempo; ni se ha producido en las propias mujeres de las comunidades un proceso de movilización, por ejemplo en contra de la violencia que ven o padecen, y haciendo un ejercicio preliminar de lo que sería una sociología de las ausencias (DE SOUSA SANTOS, 2011) en el feminismo cubano de hoy ¿podríamos realmente hablar de una práctica política y una lucha feminista en Cuba que nos representa a todas?

La experiencia etnográfica de una vida personal y académica en las márgenes me han hecho sentir/plantear desde hace tiempo ya, siguiendo a Donna Haraway, que:

La objetividad feminista trata de la localización limitada y del conocimiento situado, no de la trascendencia y el desdoblamiento del sujeto y el objeto. (...) El feminismo trata de las ciencias del sujeto múltiple con (como mínimo) doble visión. (...) trata de una visión crítica consecuente con un posicionamiento crítico en el espacio social generizado no homogéneo (HARAWAY, 1995, p. $327 ; 336)$.

A mi juicio, deviene fundamental resignificar la lucha feminista y los Estudios/Teorías de Género en Cuba; construir epistemologías que nos identifiquen, no porque la necesidad sea diferenciarnos por mera autocomplacencia, sino por ajustar nuestras comprensiones a las realidades que estudiamos en una nación bien singular con respecto al mundo, con respecto incluso a nosotros mismos en un devenir histórico, dialéctico y contradictorio de cambios y transformaciones. De igual manera, urge desmontar el formalismo funcional de muchas estructuras, entre las cuales se encuentra hoy la FMC, e intentar construir una nueva práctica política feminista de lucha, más acorde con los tiempos que corren.

\section{El problema de la representación: los estudios en Cuba y el caso de Moa}

Lejos de La Habana y las capitales provinciales cubanas, el sujeto del feminismo - o del posible feminismo - está ubicado básicamente en el accionar de la FMC. Y es un sujeto, así en singular, porque no existe una multiplicidad real de voces ni sujetos del feminismo. Sin embargo, las investigaciones que he desarrollado sobre violencia de género en contextos rurales, suburbanos y urbanos, como Moa y Sagua de Tánamo en la provincia de Holguín (HERNÁNDEZ, 2013a; HERNÁNDEZ, 2013b; HERNÁNDEZ, 2014a; HERNÁNDEZ; DELGADO, 2014b); así como los trabajos de otras investigadoras del Núcleo de Estudios de Género de la Universidad de Holguín en el oriente cubano (María de los Ángeles ARIAS, 2013; Ania PUPO, 2016), han puesto al descubierto cuestiones que hablan en detrimento de tal accionar de la FMC y de la necesidad de resignificación en la práctica política feminista (académica o activista) en Cuba, de la que he estado reflexionando, más allá de las citadinas fronteras de la capital nacional y las provinciales. En este sentido, considero fundamental ilustrar el análisis con mis resultados del Estudio de Caso de Moa durante más de diez años, sobre distintas problemáticas de la violencia de género.

Moa, municipio de la provincia oriental ${ }^{4}$ de Holguín, fue uno de los espacios con la intención de ser convertidos en luminosos ${ }^{5}$ (SANTOS, 1996) dentro de las políticas de desarrollo del país a través de la inversión en la industria cubana del níquel. Sin embargo, a casi tres décadas del inicio de la crisis socioeconómica que impactó a la nación, Moa exhibe un deterioro claramente visible en casi todos los aspectos de la vida social. Es un contexto minero, donde, a pesar de los servicios disponibles para todas las personas en Cuba relativos a salud, educación, ciertos ámbitos de la cultura, la recreación, entre otros, la calidad de vida de sus pobladores es inadecuada. La contaminación ambiental destruye con gran fuerza la infraestructura habitacional privada y todo tipo de construcción, sin que existan posibilidades reales de mantener, reparar y conservarlas, a la vez que afecta seriamente la salud. El desarrollo social es cuestionable debido a que, entre muchos factores, la alta contribución económica que aporta la industria del níquel al país no se

\footnotetext{
${ }^{4}$ Ser oriental y todo Oriente en Cuba está cargado de un simbolismo y una retórica de la inferioridad, ya sea social, personal, políitica, cultural, económica, y otros estigmas.

${ }^{5}$ Como he argumentado en otro trabajo (HERNÁNDEZ, 2014a), a pesar de los adelantos para varios territorios de Cuba a través de las distintas políticas y acciones de Gobierno a nivel nacional, en otros estas han tenido a largo plazo consecuencias paradójicas en la medida en que estas políiticas fueron aplicadas sin tener en cuenta que se partía de niveles de desarrollo socioeconómico y cultural diferentes y desiguales en todo el país. El resultado de esas acciones lo evidencian los estudios de desigualdades sociales que han sido posteriores a los años noventa del siglo XX, en los cuales han sido evaluados los impactos de la crisis económica, sobre todo las regresiones a estados de precariedad de algunos grupos sociales, como por ejemplo las mujeres campesinas orientales o las mujeres divorciadas con bajos ingresos y varios hijos, que ya acumulaban arrastres desde el comienzo de cambios generados por la Revolución.
} 
revierte en desarrollo social, económico y cultural hacia el interior del territorio. De igual forma, ante la carencia de opciones para invertir sanamente el tiempo de ocio, constituyen elementos de la vida social en Moa el alcoholismo, la violencia social y de género, la corrupción, la ausencia de compromiso social y otros (HERNÁNDEZ; DELGADO, 2014b).

Como los datos cuantitativos cobran una importancia fundamental para tener nociones sobre la magnitud de la ocurrencia de los fenómenos, los datos sobre violencia contra las mujeres por motivos de género que he recogido en Moa desde el 2002 arrojan estadísticas elevadas de mujeres víctimas (HERNÁNDEZ, 2014a). Estas estadísticas, que no se hacen públicas en ninguna parte, muestran un promedio anual de 155 denuncias en la Policía Nacional Revolucionaria (PNR) hasta 2017 (HERNÁNDEZ, 2017), con una tendencia estable por muchos años, además de varios subregistros por problemas de recogidas de las informaciones. Este dato se valora como elevado para un municipio, de acuerdo con los resultados de otros estudios y publicaciones sobre el problema en el país, en especial los de la Dra. Proveyer (2014).

En la investigación doctoral, específicamente de 2010 a 2014, trabajé con 47 mujeres reconocidas por la Policía como víctimas de violencia. De ellas, 6 con instrucción universitaria, 9 con técnico medio, 13 con secundario, 17 con preuniversitario y 2 con formación de nivel primario (una de ellas proveniente de la Educación Especial); es decir, la mayor cantidad de mujeres investigadas tenían una formación académica baja. De igual forma, predominaron las mujeres mestizas, y ello fue un resultado aleatorio, no intencionado. La mayoría ( 29 mujeres) eran creyentes en Dios. En cuanto a sus actividades, 27 eran trabajadoras asalariadas y 20 amas de casa; todas entre 21 y 50 años de edad. De ellas, 41 mantenían aún la relación de pareja con el victimario y solo 6 habían logrado separarse. Gran parte eran provenientes de familias donde se vivía la violencia física contra las mujeres, o con interacciones muy patriarcales y roles de género rígidos. Solo 1 de las 47 mujeres no tenía hijos.

Otra cuestión importante es que casi la mitad de las mujeres estudiadas vivían además en condiciones de precariedad habitacional, de conjunto con la económica en tanto pocos recursos económicos propios, y dependían de sus parejas. Los espacios geográficos en los que residían (con mayor presencia de lugares rurales y sub-urbanos), estructuralmente presentan una variedad de realidades, así como los más altos índices de denuncias de violencia contra mujeres, con una característica fundamental predominante en ellos: la opacidad (SANTOS, 1996), constituida por insalubridad, contaminación ambiental, inexistencia o carencia de alumbrado público, dificultades con la pavimentación de calles, problemas con acceso al agua potable, economía informal, hacinamiento, precariedad en los fondos habitacionales, bajos salarios, desempleo juvenil, desempleo femenino y masculino, marginalidad y delincuencia social, todo lo cual ha identificado a estos espacios como de riesgo, altos niveles de alcoholismo y violencia social, disfuncionalidad familiar y de las instituciones sociales y organizaciones que existen en ellos (HERNÁNDEZ; DELGADO, 2014b).

Desde un plano cualitativo, en las investigaciones han emergido múltiples problemáticas, algunas que a mi juicio cobran una alta significación: en poblados rurales he identificado prácticas de violencia contra mujeres como el arrastre por caminos de piedra o tierra y tiradas por el pelo, mujeres puestas a correr delante de todos perseguidas con cuchillos y violación sexual incestuosa a niñas y adolescentes; en barrios suburbanos y marginales, mujeres amenazadas y/o golpeadas con machetes a la vista de todos, así como otras apuñaladas en el portal y arrastradas hacia adentro de la casa por el cabello.

Toda esta violencia tiene un correlato simbólico de disciplinamiento de género: he identificado casos en los que la violencia física adopta la forma de castigos físicos intencionados, públicos, exhibidos-exteriorizados, con connotación de espectáculo, que emergen como un texto de la cultura patriarcal y que adopta en ciertos momentos, rasgos rituales. Este tipo de castigos a mujeres en los estudios realizados en Moa, ha sido documentado en prácticas de violencia de la Edad Media (Michael FOUCAULT, 1995); incluso hoy en Latinoamérica, África y otros lugares, aunque ello en Cuba no esté asociado a los males sociales que he mencionado anteriormente en este trabajo.

Acerca de la violencia sexual contra niñas y adolescentes (más que los niños), he identificado múltiples casos de violación incestuosa de padres y abuelos a hijas y nietas que obligan a establecer relaciones de pareja. En estos se han instituido pactos de silencio que determinan denuncias mucho tiempo después de la primera ejecución, en las cuales los Jefes de Sector de Policía han jugado el rol fundamental. El análisis de esos resultados y la continuidad actual de la investigación me conducen a pensar que, como en la violencia contra las mujeres, la que se produce contra niñas, niños y adolescentes también puede estar constituyendo solo la punta del iceberg y el subregistro existente de casos reales estar sobrepasando lo imaginado (HERNÁNDEZ, 2017).

Deteniendo la mirada y el análisis en este fenómeno ante el gran volumen y variedad de denuncias en cuanto al tipo de delitos contra menores existentes en la Policía, analicé un primer 
período de 6 años escogiendo los delitos con mayor presencia en las denuncias (violación sexual -incluyendo las practicadas mediante el incesto- y abuso lascivo), para contrastarlo con los datos a nivel del país, publicados en solo dos Informes oficiales, uno de 2013 y el otro de $2015^{6}$. Los datos y su comprensión estadística (HERNÁNDEZ, 2017) mostró (Ver Tabla 1 y Gráfico 1).

Tabla 1 - Datos violencia contra menores em Moa. Período 1-1-2010 al 30-12-2015

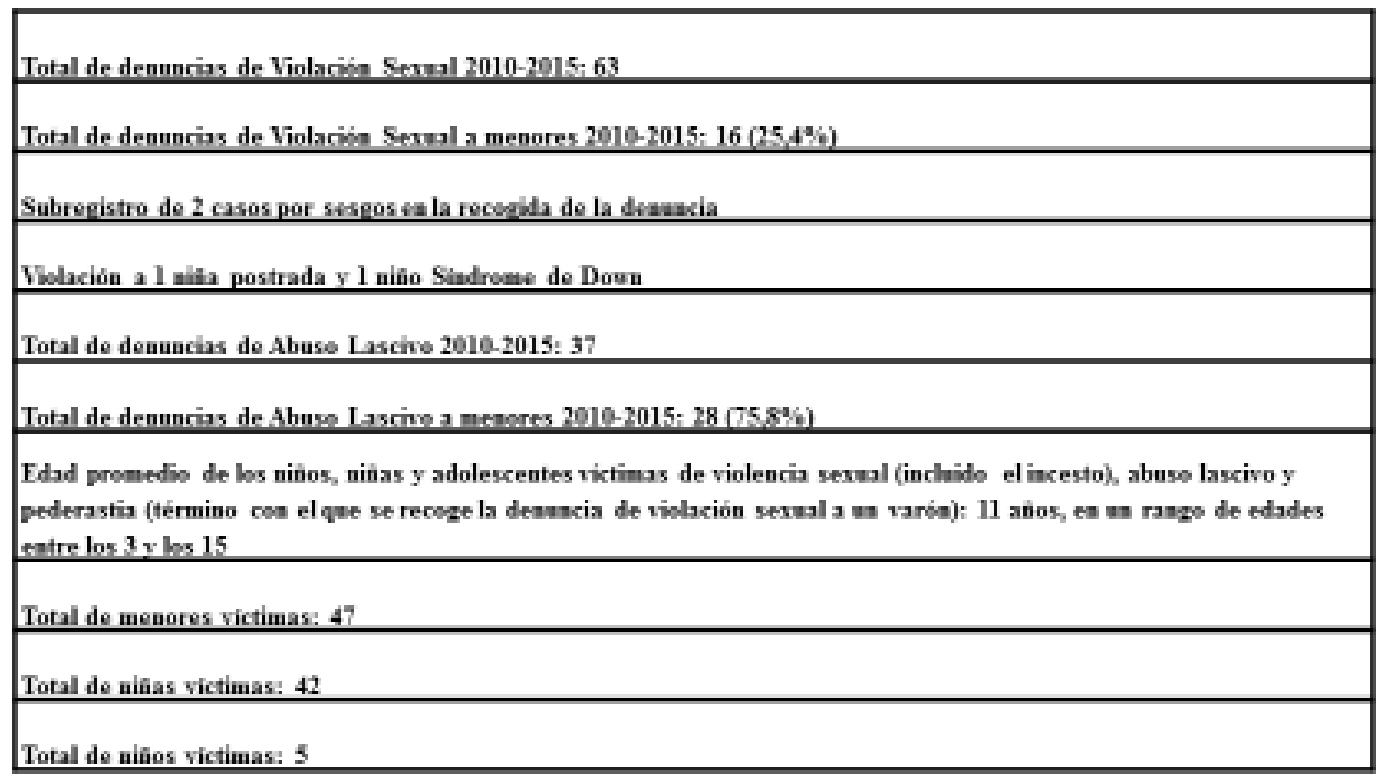

Gráfico 1 - Datos violencia contra menores em Moa. Período 1-1-2010 al 30-12-2015

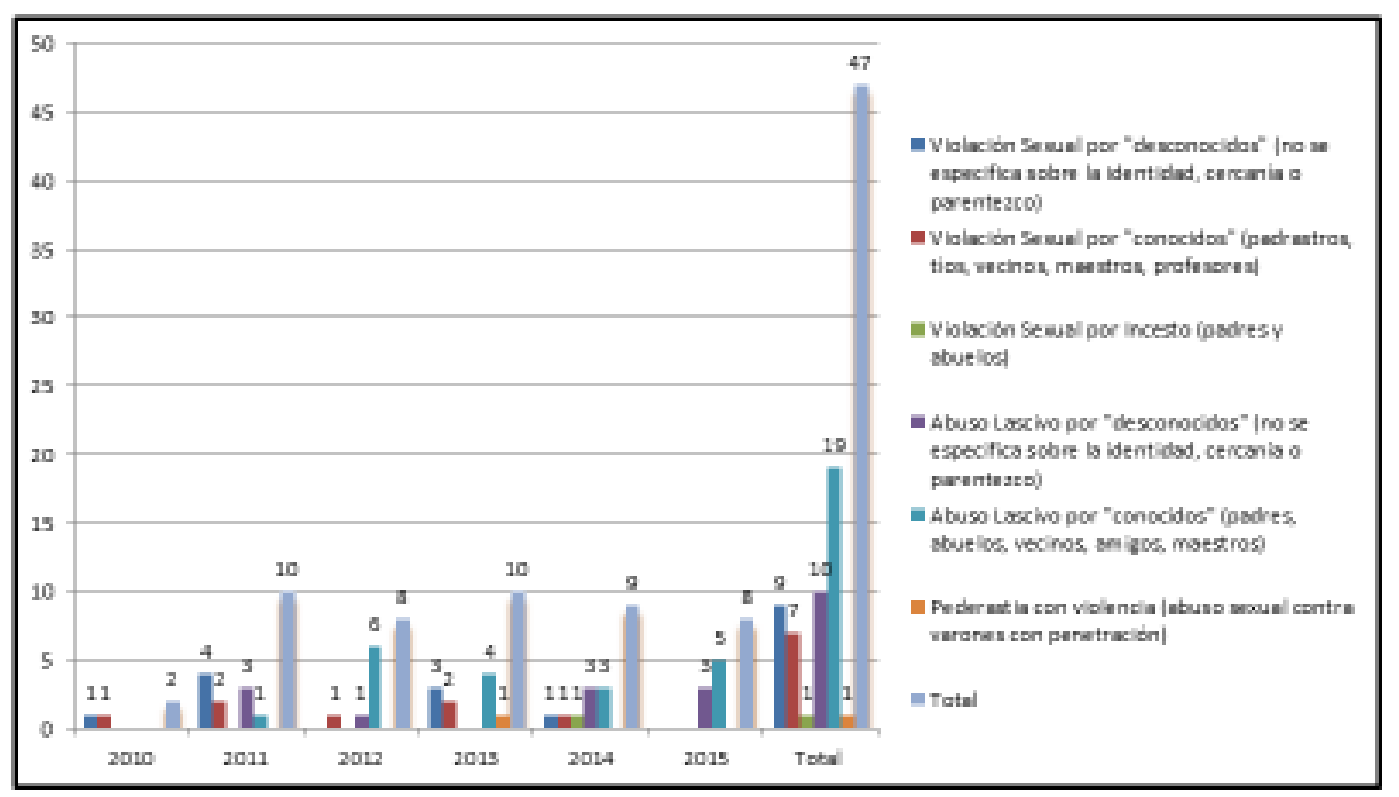

Fuente: Denuncias radicadas em la PNR. Moa. 2010-2015

\footnotetext{
${ }^{6}$ Estos Informes fueron encontrados en Internet, en: Pérez Peña, Yamilet: Cuba ratifica compromiso internacional contra la trata de personas, Cubadebate, 2013. Disponible en: http://www.cubadebate.cu/noticias/2013/10/15/ cuba-no-permite-la-trata-de-personas-ni-la-explotacion-sexual/\#.WDcIE3uNMd4. Acceso el: 25/09/2016; y Tesoro, Susana: Parlamentarios cubanos evalúan situación de niños víctimas de abuso, Cubadebate, 2016. Disponible en: http://www.cubadebate.cu/noticias/2016/07/07/parlamentarios-cubanos-evaluan-situacion-de-ninos-victimas-de-abuso/ \#.WDclkHuNMd4. Acceso el: 25/09/2016.
} 
En una posterior indagación realizada en aras de analizar las tendencias, del 1 ro de enero de 2016 a 30 de mayo de 2017, identifiqué 17 nuevos casos de denuncias en la Policía de violencia sexual y abuso lascivo contra niñas, niños y adolescentes (Ver Tabla 2 y Gráfico 2).

Tabla 2 - Datos violencia contra menores em Moa. Período 1-1-2016 al 30-5-2017

\begin{tabular}{|c|c|c|c|}
\hline Año/Tipo de Delito & 2016 & 2017 & Total \\
\hline \multicolumn{4}{|l|}{ Yuolación Sexnd por "desconocidos" (no se expecifica sobre la identidad, cercaria oparentesco) } \\
\hline $\begin{array}{l}\text { Volación Sexul por "conocidos" (padrastros, tos, vechos, maestros, profesores, novios, ex novios, } \\
\text { prodicadores, coros conocidos) }\end{array}$ & 2 & & 2 \\
\hline Yiolación Sexud por Incesto (padres y abuelos) & 3 & & 3 \\
\hline Abuso Lawcho por "desconocidos" (no se espoctica sobre h identidad, cercania oparentesco) & 3 & 1 & 4 \\
\hline 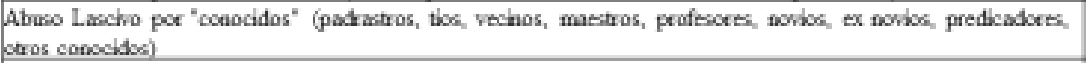 & 4 & 4 & 8 \\
\hline \multicolumn{4}{|l|}{ Pederastia con viokncia (sbuso sexusal contra varones con penetraciön) } \\
\hline Iotal & 12 & 5 & 17 \\
\hline \multicolumn{4}{|l|}{ Iotal de demunciss de Violación Sevual 2016: 8} \\
\hline \multicolumn{4}{|l|}{ Total de denunciss de Vidación Sevual a menores 2016: 5 (62.59\%) } \\
\hline \multicolumn{4}{|l|}{ Iotal de deruncias de Violsción Serual al 30 de mayo de 2017:1 } \\
\hline \multicolumn{4}{|l|}{ Iotal de derunciss de Violsción Sevusl a menores al $30 \mathrm{de}$ mavo de 2017: 0 } \\
\hline \multicolumn{4}{|l|}{ Iotal de deruncias de Abuso Lascivo 2016: 8} \\
\hline \multicolumn{4}{|l|}{ Iotal de deruncias de Abuso Lascivo a menores $2016 \times 7(87.590)$} \\
\hline \multicolumn{4}{|l|}{ Total de deruncias de Abuso Laxcivo al 30 de maro de $2017: 5$} \\
\hline \multicolumn{4}{|l|}{ Total de demuncias de Abuse Lascivo a menures al 30 de mavo de 2017: 5 (10090) } \\
\hline \multicolumn{4}{|l|}{ Abuso lascivo a un varón de 9 años } \\
\hline \multicolumn{4}{|l|}{ Total de hechos. Periodo 1-1-2016 al 30-5-2017:22 } \\
\hline \multicolumn{4}{|l|}{ Iotal de hechos contra menores. Periodo 1-1-2016 al 30-5-2017:17 (77.27\%) } \\
\hline \multicolumn{4}{|l|}{ Fdad promedo de los menores victimas de abusos lascivos (de los que estin repistrados): 9.11 abios } \\
\hline Porcierto de victimas rifixs con respecto al total de estos dos defitos & 759 & $8333 \%$ & $77.27 \%$ \\
\hline $\begin{array}{l}\text { Aumento en } 4 \text { casos en } 2016 \text { con respecto a 2015. Y hasta la fecha de estudio, amento en } 9 \text { casos, duplicindose } \\
\text { In cifin mis mo. }\end{array}$ & & & \\
\hline
\end{tabular}

Gráfico 2 - Datos violencia contra menores em Moa. Período 1-1-2016 al 30-5-2017

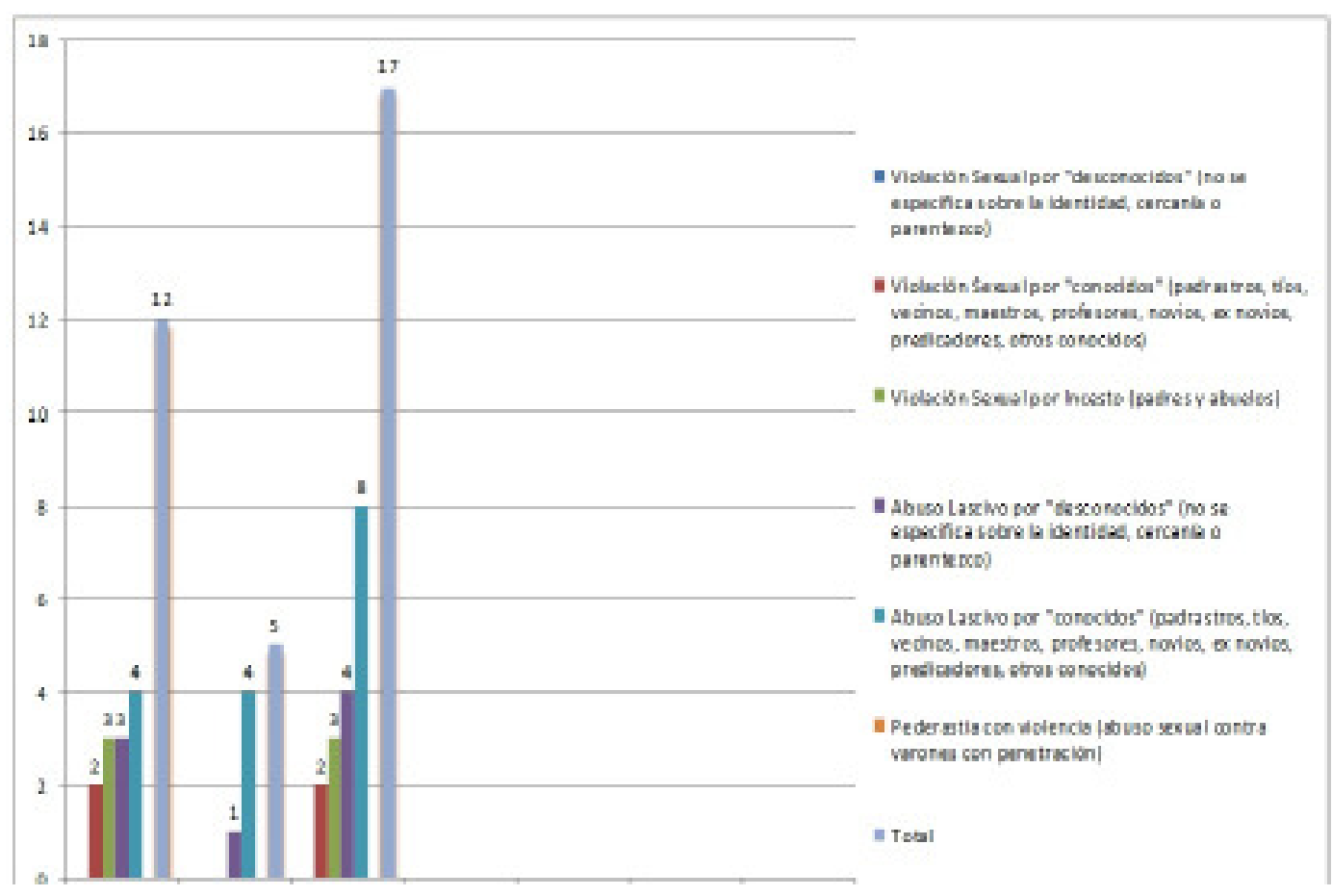

Fuente: Denuncias radicadas em la PNR. Moa. 1-1-2016 a 30-5-2017

En estas prácticas de violencia sexual contra menores son tocados sus órganos sexuales, introducidos los dedos, realizadas succiones y marcas en el cuerpo, son besados y manoseados los senos de las niñas y adolescentes. Los espacios geográficos en los que se han producido, continúan siendo fundamentalmente los rurales y sub-urbanos. En este período, la edad promedio de los niños, niñas y adolescentes víctimas disminuyó de 11 años a 9.

Un dato significativo es que mientras en el país la edad promedio de las niñas, niños y adolescentes víctimas de violencia sexual se ubica entre los 13 y los 15 años, en Moa la edad 
promedio de este grupo es menor. De igual forma, mientras en los victimarios de los Informes nacionales las edades oscilan entre los 18 y los 30 años, en Moa los rangos de edades van desde los 30 años hasta los 72; lo que significa que aquellos que atentan sexualmente, son personas de mayor edad en contraste con edades más tempranas de las víctimas. En tales Informes, los lugares donde más se producen estos hechos son los espacios de concentración pública; mientras en Moa, la mayor cantidad de denuncias se produce sobre casos ocurridos en las zonas rurales (de poca concentración habitacional) y sub-urbanas (más cerca del sentido de marginalidad socioterritorial, exclusión y carencias, Milton SANTOS, 1996).

Para realizar una comparación válida con los informes publicados a nivel nacional, se consideró imprescindible tomar los mismos años bases de cálculo a los cuales se hace referencia en los mismos. Analizando los años 2012 y 2015 (tal y como se presentan los datos a nivel nacional), se observa que en Moa se produce una tendencia al crecimiento relativo de las víctimas con respecto al porciento promedio anual propio (0,044): de 0,045 en el 2012 crece a 0,047 en el 2015, cuando en el país la tendencia ha sido al decrecimiento del fenómeno de un 0,09 al 0,001\% respectivamente. Para 2017, el crecimiento respecto a 2015 es del 50\%, con una tasa de 0-16 años que alcanza un 0,067 (HERNÁNDEZ, 2017).

El análisis del total de estos datos implica identificar una cifra estadística superior al porciento medio en el país, en especial en el año de comparación 2015: el 0,047 \% de Moa es 47 veces superior a la cifra de niñas, niños y adolescentes víctimas de violencia sexual con respecto al dato nacional de 0,001 \%, visto como frecuencia relativa, donde 0,047 / 0,001 $=47$. Si el porciento de casos del país se ha mantenido, pues hasta el momento no hay informe publicado, en Moa hasta la fecha de análisis en mayo de 2017 se han producido 67 casos más que la media nacional, en lo que se identifica un crecimiento exponencial (Ver Gráfico 3).

Gráfico 3 - Datos totales violencia contra menores em Moa. Período 1-1-2010 al 30-5-2017

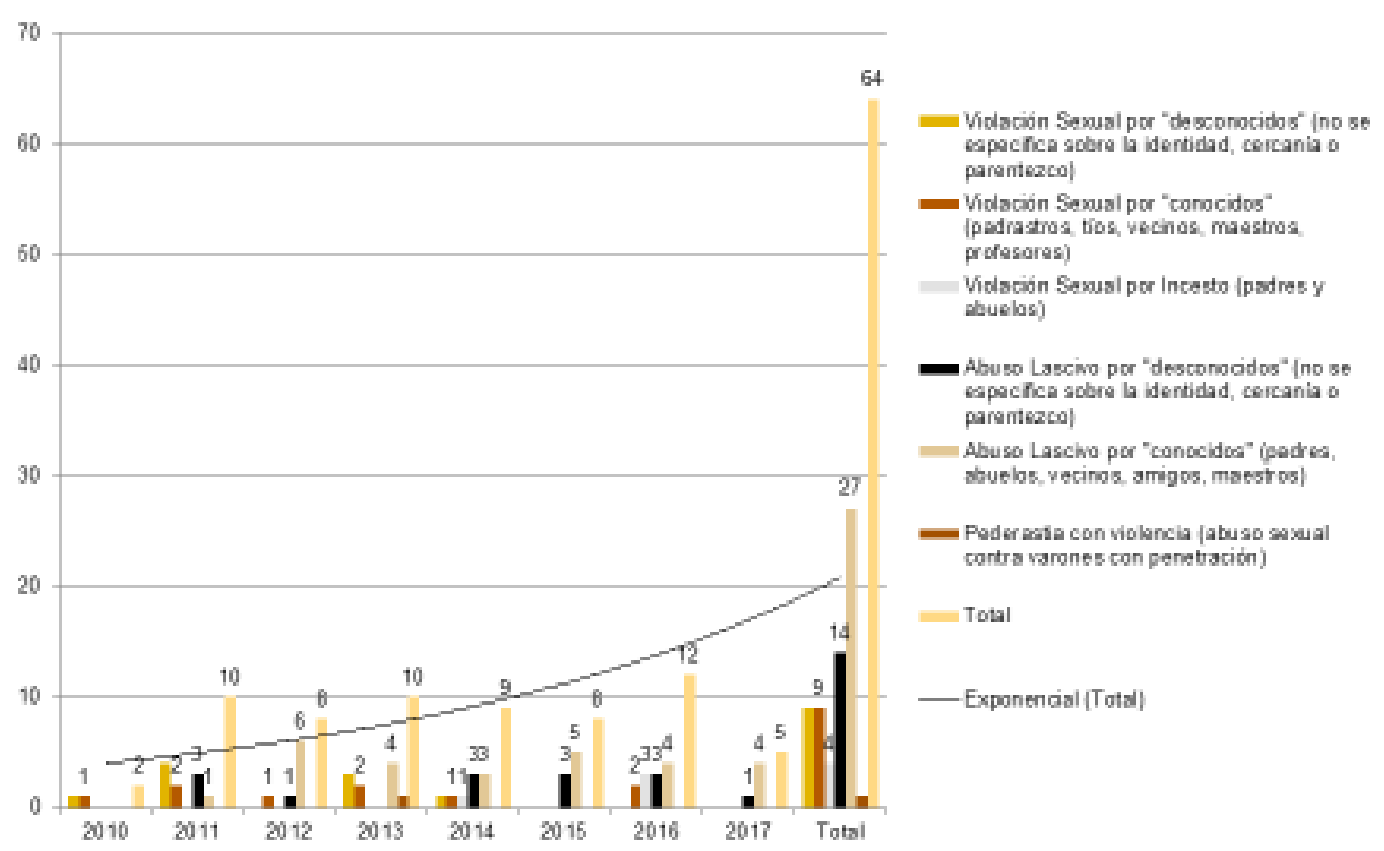

Fuente: Denuncias radicadas em la PNR. Moa. 1-1-2010 a 30-5-2017

Estos datos muestran que no obstante el fenómeno de la violencia sexual contra niñas, niños y adolescentes en Cuba no sea considerado un problema social tras lecturas cuantitativas, es un fenómeno que existe y se halla tras el silencio de la cultura patriarcal aún cotidiana, de las familias y de las cifras institucionales visibles. Desde un análisis feminista descolonial, es un fenómeno marcado por lo territorial, la pobreza, lo marginal, lo suburbano y lo rural; por el hacinamiento habitacional, las escasas posibilidades de acceso a rutas críticas de atención a la violencia de género; por la vulnerabilidad ligada a la edad y a las clases sociales bien diferenciadas hoy en Cuba. Los estudios realizados en Moa evidencian que son víctimas en contextos de familias y comunidades con muy bajos recursos económicos, rozando el límite de la pobreza cruda de muchos países del mundo?.

\footnotetext{
${ }^{7}$ Sobre este tema de la pobreza en Cuba hoy, puede verse en Internet los debates de la Revista cubana Temas.
} 
De manera general, la investigación sobre violencia contra mujeres, niñas y adolescentes por motivos de género en Moa, me remite a pensar, fundamentalmente, en los análisis de dos autoras feministas descoloniales de América Latina: Rita Segato y Karina Bidaseca. Las prácticas exhibitorias de disciplinamiento, de inscripción y escritura en el cuerpo de las mujeres, niñas y adolescentes de la violencia de género que he llegado a conocer y estudiar en Moa, los pactos de silencio, el efecto simbólico de hermanamiento entre hombres de comunidades y espacios geográficos específicos, singulares por cuanto imprimen distintividad con respecto a otros espacios, y que no forman parte de los escenarios investigativos del feminismo académico dominante, me conducen a valorar que, aunque en Cuba las bases del fenómeno no sean las mismas de lo que se denomina justamente femi-genocidio, comparten un común orden patriarcal de intensidad que se va elevando en la medida en que nos vamos transformando como país en otra cosa distinta a como inició la Revolución, compartiendo ya hoy - en mucho - rasgos y prácticas del capitalismo que tanto se convirtió en nuestro enemigo histórico. Hay muchas interseccionalidades que deben ser profundamente indagadas en Cuba: un espacio diverso, aún en crisis de reformulación socio-económica y política, pero que sigue defendiendo el ideal de una sociedad más justa para todos y todas. Segato:

Esto que sucede en Moa, tiene que ver con lo que plantea Bidaseca (2015), siguiendo a

Otras experiencias de violencias que conocemos, ocurren en esos sitios glolocales en que las mujeres están siendo utilizadas en las guerras difusas; no como el fin sino como el medio, como "dadoras del tributo por el cual se reconûrma la hermandad viril», en palabras de la antropóloga Rita Segato.(...) se trata de un problema social del dominio de las relaciones de poder y dominación, por medio del cual se establece una jerarquía de valores que otorga al género masculino la superioridad por sobre el femenino; en el contexto de la conûguración extractivista y depredadora del capitalismo en su fase contemporánea. Se trata así de pensar la analogía entre los feminicidios con el orden capitalista-masculino, racista y patriarcal - sobre los territorios - cuerpo/femenino - (BIDASECA, 2015, p. 19; 21).

¿Cuál es el propósito de tales grados de crueldad en la violencia contra las mujeres, y en los actos específicos de violación?

Uso y abuso del cuerpo del otro sin que éste participe con intención o voluntad compatibles, la violación se dirige al aniquilamiento de la voluntad de la víctima, cuya reducción es justamente significada por la pérdida del control sobre el comportamiento de su cuerpo y el agenciamiento del mismo por la voluntad del agresor. (...) debido a la función de la sexualidad en el mundo que conocemos, ella conjuga en un acto único la dominación física y moral del otro (SEGATO, 2013, p. 20, 21).

Sin embargo, ante toda esta realidad, en las investigaciones he podido identificar que en el trabajo del Gobierno local y en el de la FMC en Moa no existe una percepción adecuada de la violencia de género como problema social, y desconocen las magnitudes cuantitativas de la problemática. Todas las mujeres víctimas con las que he trabajado expresan no creer en la efectividad de la FMC para ayudarles con su problema y sentirse desamparadas. El resultado es que no hay transformación social positiva y hay que comenzar a pensar en la violencia sexual contra menores. A esto se suma que no existen mecanismos oficiales que permitan un respaldo material a las necesidades que pudiera identificar la FMC para enfrentar la violencia contra las mujeres como problema social. En ese escenario, no obstante las dificultades en su trabajo, solo el accionar de la Policía y sus Sectores devienen instituciones que han representado algún grado de protección para estas mujeres, lo cual ha sido posible comprender desde el discurso de la mayor parte de ellas (HERNÁNDEZ, 2017).

Hoy, en mi contexto de estudio, la violencia física contra las mujeres por motivos de género tiene una carga de crueldad significativa, nada despreciable con respecto a los estudios de Segato (2013) en Ciudad Juárez. Las muchas e intensas amenazas de muerte a las que se ven sometidas, tras contundentes castigos corporales en base a heridas con cuchillos y machetes, patadas, fracturas y lesiones de partes del cuerpo como brazos, costillas, clavículas, piernas y cráneo; la triste realidad de que las amenazas menos graves que no causan peligro inminente para la vida de las mujeres están antecedidas igualmente de machetazos, de golpes contundentes con los puños y fracturas en el cuerpo, de heridas con cuchillos y puñales, que en más del $90 \%$ de los casos son resueltos con multas administrativas equivalentes a poco más de un dólar estadounidense y que no se convierten en delito, sin detenerme en analizar la violencia psicológica, la económica, la estructural, u otras tantas posibles, me llevan a interrogarme ¿dónde está hoy el resultado de nuestras luchas históricas y presentes? ¿quién o quiénes están viendo estas otras violencias de género? ¿cómo saber que esto no están ocurriendo -o no sean una regularidad hoy de la violencia de género-en otros espacios que se escapan a la vista del sujeto de la enunciación actual del feminismo en Cuba, en especial del feminismo académico, ubicado en espacios distantes de la vida cotidiana de mujeres distribuidas en un bastante amplio espacio geográfico 
nacional? ¿cuál es la práctica política y humana de ese sujeto del feminismo cubano posRevolución -ese que debería estar ubicado en la FMC en Cuba-más allá de las fronteras de la capital nacional y las provinciales, acá, donde no hay feminismo académico más allá de unas pocas voces para enunciar; donde no hay activismo en las redes sociales, ni activismo dentro de las propias mujeres, atravesadas por mucho más que el patriarcado: por ejemplo, la pobreza, la raza, la orientación sexual, los bajos niveles de instrucción hoy, la cadena de cuidados que están viviendo, el trabajo doméstico no remunerado, la crisis económica del país, lo espacial, lo territorial en desventaja con respecto a capitales provinciales o nacional? ¿hasta dónde nos representan los discursos nacionales del feminismo que se gesta en las urbes, ya sea en la academia o en el activismo social, incluso en el accionar propio de la FMC actualmente? ¿podríamos realmente hablar de una práctica política feminista en Cuba, y ello significa que vaya más allá de las fronteras de las capitales donde se materializa el feminismo cubano activista o académico hegemónico, que es urbano, citadino, metropocéntrico en sus estudios y acciones políticas?

Todo este panorama tiene, a mi juicio, una lectura de fondo: hoy las teorías y las metodologías que sustentan la investigación y el accionar político alrededor de la violencia de género en Cuba y del feminismo en sí mismo, se hallan basados en análisis nor-eurocentrados y extractivistas, cuando en realidad lo que se necesita son otras formas de pensar los problemas sociales y su transformación. En palabras de Rufer:

Es fundamental para pensar en metodologías otras o metodologías horizontales, asumir que los procesos sociales son generados por sujetos que no esperan certificaciones del mundo académico ni de la intelectualidad de un país; que en muchas ocasiones el lugar construido del/la investigadora es cuestionado por quienes resisten o actúan políticamente (RUFER, 2017, p. 3).

\section{A modo de conclusiones. Otras lecturas posibles}

Aunque es complejo el panorama de la violencia de género y el accionar del feminismo en Cuba/Moa hoy, existen alternativas de transformación posibles, tanto para la academia como para la práctica política. En una de ellas se colocan, precisamente, las Epistemolgías del Sur con sus procedimientos y principios; así como la aplicación de metodologías de investigación y teorización no extractivistas, que pueden ofrecernos otras lecturas, análisis y procesos de cambios.

Como bien plantea De Sousa:

Las Epistemologías del Sur reflexionan creativamente sobre esta realidad para ofrecer un diagnóstico crítico del presente que, obviamente, tiene como su elemento constitutivo la posibilidad de reconstruir, formular y legitimar alternativas para una sociedad más justa y libre (DE SOUSA SANTOS, 2011 , p. 14). (...) son profundamente históricas, pero parten de otras historias que no son precisamente la historia universal de occidente. Hay otras historias más allá de la historia de occidente, y esas historias son las que constituyen el trabajo presente y futuro de las Epistemologías del Sur (DE SOUSA SANTOS, 2011, p. 17).

En ese sentido de alusión a la historia más allá de la historia de occidente, el enfoque de las Epistemologías del Sur, en el que cobran fundamental importancia los Feminismos Descoloniales, es crucial analizar el vínculo entre la violencia de género hoy en Cuba y el problema de la representación, cuando el feminismo (activista o académico) es hegemónicamente urbano, citadino, metroprocéntrico. Siguiendo a De Sousa (2011), hay historias de vida detrás de la violencia contra las mujeres, niñas y adolescentes, voces subalternas que no se escuchan y que guardan sus heridas desde la otredad alejada. Es por ello que insisto en la necesidad de hacer más visibles en Cuba las miradas y las prácticas desde los contextos y validar la perspectiva de los actores sociales situados, del conocimiento que es siempre conocimiento parcial y situado.

A partir de estas premisas y de las problemáticas anteriormente expuestas del caso de Moa y del país en toda su extensión, considero que el estudio de la violencia de género en Cuba, en aras de realizarse más adecuadamente, debe gestarse desde metodologías no extractivistas y un nuevo feminismo; nutrirse de los presupuestos teóricos y metodológicos de tres cuestiones fundamentales que no pueden obviar las (OS) académicas (OS) y activistas feministas cubanas (OS) en este momento histórico que vivimos, cada vez más complejo, en el que se vuelven más sofisticadas las interrelaciones entre capitalismo-colonialismo-patriarcado:

1. el feminismo transnacional, en especial los análisis de Mohanty (2008) muy bien interpretados por Marta Sierra (2017) y ello significa que: en primer lugar, el feminismo no puede pensarse fuera de la colonialidad del poder, ya que el feminismo como disciplina ha sido dominado por un colonialismo discursivo y epistémico. En segundo, que es imposible pensar el género sin tener en cuenta las variantes de raza, clase social, nacionalidad, entre otras. Una discusión exclusivamente centrada en el género no hace más que reducir la práctica feminista. En tercero, que es necesario historizar y contextualizar las formas que asumen las relaciones de género para evitar el universalismo feminista. Ello conduciría a visibilizar los silenciamientos por ejemplo, del feminismo latinoamericano y las contribuciones del feminismo negro e indigenista. 
En cuarto lugar, que el concepto de cultura es un concepto que el feminismo necesita discutir junto con el género. Es necesario considerar la cultura como un proceso y no como un producto para evitar los esencialismos culturales. El feminismo entonces debe evitar dos tipos de generalizaciones: el universalismo liberal que enfatiza la igualdad que claramente representa el feminismo eurocentrista y el relativismo cultural puesto de moda por el posmodernismo y su concepción de multiculturalismo, que enfatiza la diferencia, pero la relativiza. Por último, repensar la relación geopolíica entre lo global y lo local o el llamado primer mundo y tercer mundo. La idea de localidad ha superado la unidad del estado-nación, y en este sentido es necesario pensar lo local dentro de lo global y las posibilidades que estas nuevas dinámicas ofrecen a la praxis feminista.

2. Las(os) investigadoras(es) (porque en Cuba hoy está en la ciencia feminista, su trabajo de campo, en la representación, las publicaciones, las políticas), deberían comprometerse realmente con eso que plantea Rufer:

La metodología que aquí se propone analizar busca mezclar todos los elementos que nos permitan conocer a profundidad fenómenos sociales que no serán juzgados ni sobreanalizados, sino descritos como movimientos, como procesos temporales. Entonces icómo hacer de la mirada construida en el campo una forma de episteme que no violente y no sea extractivista? Lo primero es -como ya señalé antes- tener claridad respecto a nuestro lugar de enunciación, respecto a la potencia del pensamiento como una acción política que se acompaña del soporte de la escritura. (...) la reflexividad del investigador, pensar desde dónde pensamos e imaginamos es central para cuestionar las prácticas extractivas, pero no sólo en el sentido de pensar nuestra propia etnicidad, clase y raza sino cuál es el lugar epistémico y temporal que damos a las personas con las cuales trabajamos. El problema central del extractivismo académico radica en la potencial falta de empatía y coetaneidad con el otro (RUFER, 2017, p. 3).

2. Del reto que supone -siguiendo a De Sousa con las Epistemologías del Sur-hacer en un primer momento sociología de las ausencias y ciencia feminista nacida en la lucha social, incluso en Cuba. Ello implica otras y nuevas lecturas de la realidad, de las ciencias sociales y los Estudios de Género, incluyendo en ellos y en las visiones desde las que se trabajen, los problemas de violencia; así como el carácter anticolonial, antipatriarcal y anticapitalista de estas epistemologías. Un elemento fundamental a superar en Cuba hoy desde perspectivas metodológicas no extractivistas, es la falta de compromiso de muchas (os) representantes de la academia feminista con la lucha social desde dentro, aunque eso esté mediatizado por cuestiones socio-estructurales bien complejas de analizar en el contexto de la realidad social, económica y política cubana actual.

En el amplio ámbito de estudio de la violencia contra las mujeres por motivos de género y las desigualdades en Cuba, considero clave hacer sociología de las ausencias porque esta es:

(...) la investigación que tiene como objetivo mostrar que lo que no existe es, de hecho, activamente producido como no-existente, o sea, como una alternativa no creíble a lo que existe. Se trata de transformar objetos imposibles en objetos posibles, objetos ausentes en objetos presentes. La no-existencia es producida siempre que una cierta entidad es descalificada y considerada invisible, no-inteligible o desechable. No hay por eso una sola manera de producir ausencia, sino varias. Lo que las une es una misma racionalidad monocultural (DE SOUSA SANTOS, 2011 p. 30).

En este escenario, a nivel macro y microsocial se necesitan estudios que puedan dar mayores luces sobre las situaciones de violencia de las mujeres a lo largo y ancho de la isla; así como sobre el futuro posible para las mujeres cubanas en su diversidad, traducido en mejores políticas. Pero la realización de tales trabajos, debe ir acompañada de la participación de múltiples puntos de vista, por parte de las(OS) activistas, académicas(os) cubanas(os) y las propias y reales mujeres, de forma horizontal, democrática y co-protagónica para evitar los sesgos de la representación, supuestamente objetiva, que se une a los colonialismos discursivos; así como debe ser capaz de elucidar los lugares de enunciación que tanto tienen que ver con la manera en que interpretamos los fenómenos que indagamos. Solo así, iremos construyendo nuevas visiones y soluciones a los problemas de violencia de género en Cuba hoy, precisamente desde otras perspectivas teóricas, metodológicas y políticas de nuestros sures.

\section{Referencias}

ÁLVAREZ SUÁREZ, Mayda. "Los estudios de género en Cuba: ¿dónde estamos y hacia dónde vamos?". Revista Anales de la Academia de Ciencias de Cuba, La Habana, v. 6, n. 2, p. 1-9, 2016.

ARIAS GUEVARA, María de los Ángeles. "Los debates sobre la relación entre las mujeres y el desarrollo. Aportes y críticas desde el feminismo". In: ARIAS GUEVARA, María de los Ángeles; PUPO VEGA, Ania; TORRALBAS FERNÁNDEZ, Aida (Comps.). Rompiendo Silencios. Lecturas sobre Mujeres, Géneros y Desarrollo Humano. La Habana: Editorial de la Mujer, 2013. p. 78-94. 
ASTELARRA, Judith. "De la biografía personal a la crítica teórica". In: ASTELARRA, Judith. ¿Libres e iguales? Sociedad y política desde el feminismo. La Habana: Editorial de Ciencias Sociales, 2005. p. 87-101.

BIDASECA, Karina. "Nuevas exhalaciones II. La interseccionalidad en las marcas indelebles de los cuerpos femeninos subalternos para una política de la memoria". Educación y Humanismo, Barranquilla, v. 15, n. 24, p. 38-53, 2013.

BIDASECA, Karina. Escritos en los cuerpos racializados. Lenguas, memoria y genealogías poscoloniales del feminicidio. España: Universidad de Las Baleares, 2015.

CASTRO-GÓMEZ, Santiago. "Decolonizar la universidad. La hybris del punto cero y el diálogo de saberes". In: CASTRO-GÓMEZ, Santiago; GROSFOGUEL, Ramón (Eds.). El giro decolonial. Reûexiones para una diversidad epistémica más allá del capitalismo global. Bogotá, D. C.: Siglo del Hombre Editores, 2007. p. 79-92.

CIPS. Las familias cubanas en el parteaguas de dos siglos. Colombia: D'vinni S.A, 2010.

CIPS. Violencia de género en las familias. Encrucijadas para el cambio. La Habana: Publicaciones Acuario del Centro Félix Varela, 2011 a.

CIPS. Violencia familiar en Cuba. Estudios, realidades y desafíos sociales. La Habana: Editorial del CENESEX y Publicaciones Acuario del Centro Félix Varela, $2011 \mathrm{~b}$.

DE SOUSA SANTOS, Boaventura. "Epistemologías del Sur". Utopía y Praxis Latinoamericana, Universidad Autónoma del Estado de México, a. 16, n. 54, p. 17 - 39, Julio/Sept, 2011.

DELGADO TORNÉS, Alicia Natividad; HERNÁNDEZ GARCÍA, Yuliuva. "Violencia contra las mujeres en la relación de pareja y cultura patriarcal. Una propuesta teórica de análisis desde la Sociología de la Cultura". Trabajo Social Sin Fronteras, debate intercontinental, Universidad de Colima, México, vol. 3, n. 6, p. 87-100, 2011

ESPINOSA MIÑOSO, Yuderkys. "Una crítica descolonial a la epistemología feminista crítica". EI Cotidiano, Universidad Autónoma Metropolitana Unidad Azcapotzalco, Distrito Federal, México, n. 184, p. 1-10, marzo/abril, 2014.

FOUCAULT, Michael. Discipline \& Punish. The birth of the prison. New York: Vintage Books. A division of Random House, Inc., 1995.

HARAWAY, Donna. Ciencia, cyborgs y mujeres La reinvención de la naturaleza. Universidad de Valencia: Ediciones Cátedra, 1995.

HERNÁNDEZ GARCÍA, Yuliuva. Estudio de caso sobre violencia contra mujeres en Calabazas, Sagua de Tánamo. Algunos aspectos culturales. Sexología y Sociedad, La Habana, vol. 17, n. 46, p. 4-1 1 , 2011.

HERNÁNDEZ GARCÍA, Yuliuva. "Influencia de la familia moense en la trasmisión de la violencia contra las mujeres. Estudio de casos". In: FLEITAS, Reyna; ROMERO, Magela (Comps.). Familia, género y violencia doméstica. Diversas experiencias de investigación social. La Habana: Instituto Cubano de Investigación Cultural Juan Marinello, 2013a. p. 281-290.

HERNÁNDEZ GARCÍA, Yuliuva. "Estudio de la violencia contra las mujeres en la relación de pareja. Resultados de investigaciones sociales en Moa". In: ARIAS GUEVARA, María de los Ángeles; PUPO VEGA, Ania; TORRALBAS FERNÁNDEZ, Aida (Comps.). Rompiendo Silencios. Lecturas sobre Mujeres, Géneros y Desarrollo Humano. La Habana: Editorial de la Mujer, 2013b. p. 89-103.

HERNÁNDEZ GARCÍA, Yuliuva. Lecturas culturales sobre la violencia contra las mujeres en la relación de pareja. El caso de Moa. 2014a. Tesis (Doctorado en Ciencias Sociológicas), CEDIC, Universidad de Oriente; Santiago de Cuba, Cuba.

HERNÁNDEZ GARCÍA, Yuliuva; DELGADO TORNÉS, Alisa Natividad. "El papel de la pobreza y la exclusión en la violencia contra las mujeres en Moa, Cuba". La manzana de la discordia, Universidad del Valle, Cali, v. 9, n. 2, p. 43-56, julio/dic., 2014b.

HERNÁNDEZ GARCÍA, Yuliuva. "Políticas públicas locales para atender la violencia de género en Cuba: entre desafíos y la realidad social de las mujeres víctimas". Encuentros, Universidad Autónoma del Caribe, Barranquilla, v. 14, n. 02, p. 105-120, julio/diciembre, 2016. 
HERNÁNDEZ GARCÍA, Yuliuva; DELGADO TORNÉS, Alisa Natividad. "Mujeres víctimas de violencia en Cuba. Un estudio de estrategias disciplinares, narrativas de violencia, espacios geográficos y dispositivos ineficaces". La manzana de la discordia, Cali, vol. 1 1, n. 2, p. 75-90, julio - diciembre, 2016. ISSN: 1900-7922

HERNÁNDEZ GARCÍA, Yuliuva. "Violencia de género contra mujeres, niñas y adolescentes en Moa. Estudio de caso en espacios de ruralidad, incesto y pactos de silencio". Novedades en población, Universidad de La Habana, La Habana, n. 25, p. 56-71, enero/junio, 2017.

HERNÁNDEZ PITA, Iyamira. Violencia de género. Una mirada desde la Sociología. La Habana: Editorial Científico-Técnica, 2014c.

MOHANTY, Chandra Tapalde. "De vuelta a Bajo los ojos de Occidente: La solidaridad feminista a través de las luchas anticapitalistas". In: HERNÁNDEZ CASTILLO, Rosalba Aída; SUÁREZ NÁVAZ, Liliana (Eds.). Descolonizando el feminismo: teorías y prácticas desde los márgenes. Cátedra: Madrid, 2008, p. 404- 468.

PROVEYER CERVANTES, Clotilde. "Gender violence: an approach based on the cuban reality". Sexología y Sociedad, La Habana, v. 20, n. 1, p. 3-17, 2014.

PUPO, Ania. Lecturas desde la perspectiva de género y el espacio sobre pobreza rural en el oriente cubano: el caso de Maceo, en Holguín. 2016. Tesis (Doctorado en Ciencias Sociológicas), CEDIC, Universidad de Oriente; Santiago de Cuba, Cuba.

QUIJANO, Aníbal. "Colonialidad del poder y clasiûcación social”. In: CASTRO-GÓMEZ, Santiago; GROSFOGUEL, Ramón (Eds.). El giro decolonial. Reûexiones para una diversidad epistémica más allá del capitalismo global. Bogotá, D. C.: Siglo del Hombre Editores, 2007, p. 93.126.

RUFER, Mario. "El habla, la escucha y la escritura. Subalternidad y horizontalidad desde la crítica poscolonial". In: CORONA BERKIN, Sarah; KALTMEIER, Olaf (Eds.). En diálogo. Metodologías horizontales en Ciencias Sociales. México: Gedisa, 2012, p.1.25.

RUFER, Mario. Resumen analítico de las clases 4 y 5 del Curso Metodologías Otras. 2017. Disertaciones en la Especialización en Epistemologías del Sur, CLACSO, Argentina.

SANTOS, Milton. A natureza do espaço. Sao Paulo: Editorial Hucitec, 1996.

SEGATO, Rita Laura. "Femigenocidio y feminicidio: una propuesta de tipificación". Revista Herramienta, Buenos Aires, n. 49, p. 1-10, 2012.

SEGATO, Rita Laura. La escritura en el cuerpo de las mujeres asesinadas en Ciudad Juárez. Territorio, soberanía y crímenes de segundo estado. Buenos Aires: Tinta Limón, 2013.

SIERRA, Marta. Clase 10, en el Curso Metodologías Otras. 2017. Disertaciones en la Especialización en Epistemologías del Sur, CLACSO, Argentina.

SPIVAK, Gayatri. ¿Pueden hablar los subalternos? Barcelona: Museu D'art Contemporani de Barcelona, 2009.

Yuliuva Hernández García (yuliuvahg@gmail.com; enyah@ismm.edu.cu). Doctora en Sociología, Máster en Estudios de Género, licenciada en Psicología y profesora titular del Instituto Superior Minero Metalúrgico de Moa. Recientemente ha sido becada por CLACSO, Argentina, para cursar una especialización en Epistemologías del Sur (su primera edición: 2016-2017). Cuenta con numerosas publicaciones, así como participación en eventos sobre género y violencia en Cuba y el extranjero. Además ha compartido escenarios académicos como profesora invitada dentro de este ámbito de estudios en España $(2006,2008,2009)$ y Brasil $(2015)$. 


\section{COMO CITAR ESSE ARTIGO DE ACORDO COM AS NORMAS DA REVISTA}

García, Yuliuva Hernández. "Violencia de género, feminismo y representación en Cuba". Revista Estudos Feministas, Florianópolis, v. 27, n. 1, e53719, 2019.

CONTRIBUIÇÃO DE AUTORIA

Não se aplica

\section{FINANCIAMENTO}

Não se aplica

\section{CONSENTIMENTO DE USO DE IMAGEM}

Não se aplica

\section{APROVAÇÃO DE COMITÊ DE ÉTICA EM PESQUISA}

Não se aplica

\section{CONFLITO DE INTERESSES}

Não se aplica

\section{LICENÇA DE USO}

Este artigo está licenciado sob a Licença Creative Commons CC-BY. Com essa licença você pode compartilhar, adaptar, criar para qualquer fim, desde que atribua a autoria da obra.

\section{HISTÓRICO}

Recebido em 30/10/2017

Reapresentado em 05/05/2018

Aprovado em 05/07/2018

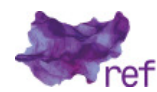

